\title{
Angiographically Documented Coronary Steal Phenomenon Evoked by the Intracoronary Infusion of Bradykinin
}

\author{
Hajime Horie, MD; Tetsuya Matsumoto, MD; Masayuki Takahashi, MD; \\ Yuko Nakazawa, MD; Hiroshi Yokohama, MD; Kazuo Minai, MD; \\ Masahiko Kinoshita, MD
}

\begin{abstract}
While studying flow-dependent coronary dilation using a Doppler flow velocity guidewire, total occlusion of a stenosed segment of the left circumflex artery during the intracoronary infusion of bradykinin was angiographically documented. Total occlusion was not demonstrated during intracoronary infusion of bradykinin after angioplasty. This is angiographic confirmation of the coronary steal phenomenon that has been previously described in the field of stress scintigraphy. (Jpn Circ J 2001; 65: 123-125)
\end{abstract}

Key Words: Bradykinin; Coronary steal phenomenon; Flow-dependent coronary vasodilation

$\mathbf{S}$ nce Keltz et al reported dipyridamole-induced ischemia, and the coronary 'steal' phenomenon as a possible mechanism, coronary steal has been emphasized in the field of myocardial stress scintigraphy. It has been defined as an increase in flow to non-ischemic myocardium with decreased flow to ischemic myocardium. However, angiographic demonstration of this phenomenon has remained elusive because of the visualization difficulty with this method. Kern et al reported a case in which steal across the stenosis was confirmed quantitatively using dual Doppler flow measurements at the proximal and distal portions of the stenotic segment during the intracoronary infusion of adenosine and intravenous infusion of dipyridamole? We observed the coronary steal phenomenon angiographically during the pharmacological hyperemia evoked by intracoronary infusion of bradykinin.

\section{Case Report}

A 71-year-old female with suspected coronary artery disease was referred to hospital for further examination. She underwent cardiac catheterization with coronary angiography (CAG) and left ventriculography (LVG). A dipyridamole stress thallium scintigram revealed reversible distribution of the lateral wall accompanied by chest pain and significant ST segment elevation after dipyridamole infusion, suggesting myocardial ischemia. Coronary angiography was performed with the Judkins technique using contrast material. Left coronary angiograms revealed significant (87\%) stenosis in the left circumflex artery (LCx); however, no significant stenotic lesions were observed in the left anterior descending artery (LAD) or right coronary artery (Fig 1). Collateral circulation to the LCx was not

(Received April 17, 2000; revised manuscript received September 13, 2000; accepted September 18, 2000)

First Department of Internal Medicine, Shiga University of Medical Science, Shiga, Japan

Mailing address: Hajime Horie, MD, First Department of Internal Medicine, Shiga University of Medical Science, Seta Tsukinowa, Otsu, Shiga 520-2192, Japan. E-mail: hajime@belle.shiga-med.ac.jp present. LVG revealed no asynergy and the ejection fraction was $74 \%$.

Combined with the positive results in treadmill testing, we considered that the stenosis in the $\mathrm{LCx}$ required interventional treatment, but prior to that we tried to assess endothelium-dependent vasodilation using intracoronary bradykinin (BK; Sigma Chemical Co, St Louis, MO, USA) in the LAD. Written informed consent was obtained from the patient before the study, which was approved by the institutional Ethics Committee. A 7F guiding catheter was positioned in the ostium of the left main coronary artery and through this a 0.014-inch Doppler flow velocity wire (FloWire, Cardiometrics Inc) was positioned in the proximal portion of the LAD. A $2.5 \mathrm{~F}$ infusion catheter was also advanced through the guiding catheter and positioned in the ostium of the left main coronary artery. Phasic and mean aortic blood pressure, heart rate, and 12-lead ECGs were continuously monitored using a Nihon-Kohden polygraph system and recorded on a multi-channel recorder. Coronary blood flow (CBF) velocity was measured continuously and peak flow velocity was determined using an on-line spectral analyzer (FloMap, Cardiometrics Inc). Peak blood flow velocity spectral signals and their mean values were monitored continuously. Mean CBF was calculated as follows: ${ }^{6}$

$\mathrm{CBF}(\mathrm{ml} / \mathrm{min})=0.5 \times$ Average peak flow velocity $(\mathrm{cm} / \mathrm{min}) \times$ Cross-sectional area $\left(\mathrm{cm}^{2}\right)$.

The cross-sectional area was determined by quantitative coronary angiography.

BK was diluted with physiological saline at a concentration of $2 \mu \mathrm{g} / \mathrm{ml}$ and sterilized at the Department of Pharmacy, Shiga University Hospital of Medical Science. It was infused at $2.0 \mu \mathrm{g} / \mathrm{min}$ for $2 \mathrm{~min}$ into the left main coronary artery through the infusion catheter using an infusion pump $(1 \mathrm{ml} / \mathrm{min})$. We had previously determined the dose at which CBF was increased to a maximal level similar to that achieved with $12 \mathrm{mg}$ intracoronary papaverine without changing systemic aortic pressure or heart rate by gradual increasing the dose from $0.2 \mu \mathrm{g} / \mathrm{min}$. Further increase in the dose of $\mathrm{BK}$ did not further increase in $\mathrm{CBF}$ and caused a 


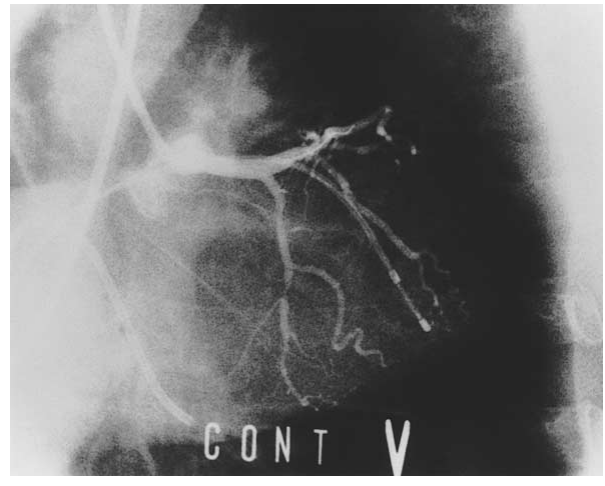

Fig 1. Cine frame in the right anterior oblique caudal projection showing significant (87\%) stenosis in the mid left circumflex artery (LCx).
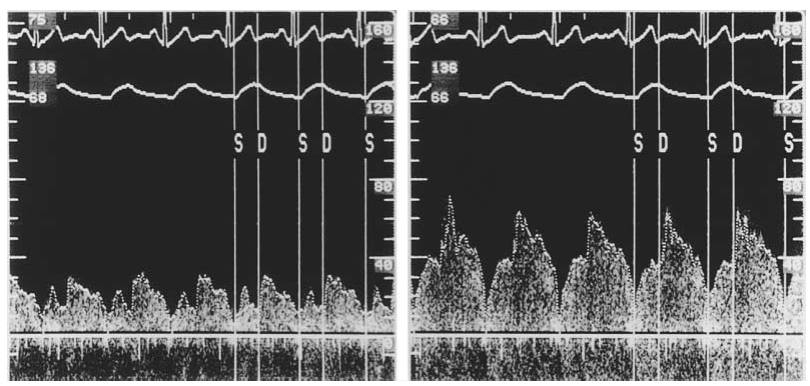

Fig 2. Flow velocity patterns in the mid left anterior descending artery (LAD) before (Left panel) and $1 \mathrm{~min}$ after (Right panel) the intracoronary infusion of bradykinin (BK).

decrease in systemic aortic pressure. One minute after BK infusion, despite a significant increase in the average peak velocity of CBF in the LAD (from $18 \mathrm{ml} / \mathrm{min}$ to $38 \mathrm{ml} / \mathrm{min}$; Fig 2) and significant dilation in the proximal portion of the LAD (from $2.0 \mathrm{~mm}$ to $2.2 \mathrm{~mm}$ in diameter), the patient complained of severe chest pain accompanied by significant transient ST segment elevation in II, III, and aVF, which was similar response as that with intravenous dipyridamole. Heart rate, mean aortic blood pressure and rate-pressure product did not change significantly during BK infusion (data not shown). Coronary angiography demonstrated total occlusion in the mid-portion of the LCx (Fig 3), with significant dilatation throughout the entire LAD. The total obstruction in the LCx and the chest pain reversed to the basal levels after intracoronary injection of nitroglycerin $(250 \mu \mathrm{g})$. Coronary angioplasty was then performed successfully. Three months later, no restenosis was seen on followup coronary angiography. The intracoronary infusion of BK was repeated with a flow-wire at the same position in the LAD as before angioplasty. Flow-dependent dilation and hyperemic flow in the LAD were reproduced during BK infusion, and the angioplasty site in the LCx was angiographically unchanged before and during BK infusion.

\section{Discussion}

It is well known that $\mathrm{BK}$, an endogenous vasoactive substance, induces endothelium-dependent relaxation of isolated human coronary artery in vitro, but recently it was reported that $\mathrm{BK}$ causes vasodilation of human epicardial coronary arteries and resistant coronary vessels in vivo. Similar to BK, dipyridamole and adenosine increase blood flow without significantly changing myocardial oxygen

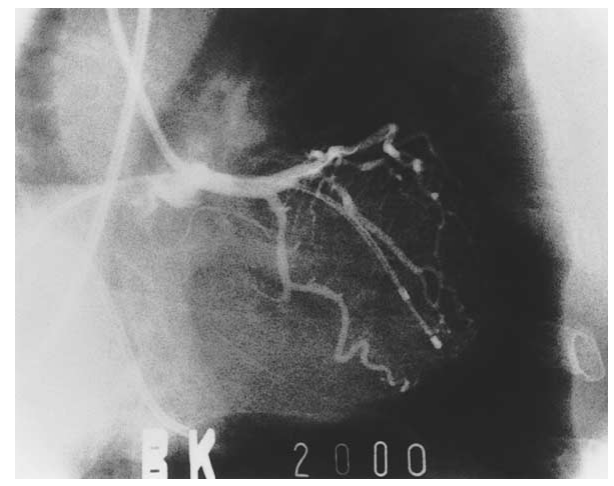

Fig 3. One minute after the intracoronary infusion of BK there is total obstruction in the mid LCx despite vasodilation in the LAD.

demand, and generally they do not produce ischemia.

In the present case, we noted epicardial coronary vasodilation and an increase in $\mathrm{CBF}$ in the LAD without any changes in myocardial oxygen demand during BK infusion. However, we documented total obstruction in the midportion of the LCx, which was significantly stenosed before BK infusion. Systemic blood pressure was unchanged during the infusion. We can not completely rule out the possibility of 'Venturi mechanism', which is related to the passive collapse of the disease-free vessel wall because of an increase in coronary blood flow velocity; however, this is unlikely because of the decreasing coronary perfusion pressure in the stenosed portion. We observed inhomogeneous myocardial perfusion without a change in coronary perfusion pressure following the intracoronary infusion of $\mathrm{BK}$ and the similar phenomenon observed after dipyridamole or adenosine infusion is considered to be coronary steal2,5 Using an intravascular Doppler guide wire, Kern et $\mathrm{al}^{2}$ quantitatively demonstrated dipyridamole-induced coronary steal phenomenon, and Voudris et $\mathrm{al}^{5}$ demonstrated coronary flow inhomogeneity induced by intravenous dipyridamole, which produced a perfusion defect on thallium-201 imaging. These 2 studies postulated the coronary steal phenomenon based on a decrease in the coronary flow velocity at peak hyperemia distal to the stenosis.

We are the first to report angiographically documented total occlusion in the mid-portion of the LCx accompanied by significant chest pain and ST elevation, as well as a significant increase in CBF in the LAD. In contrast to the 2 previous reports, we measured $\mathrm{CBF}$ in the $\mathrm{LAD}$, which is direct proof of the increase in flow to the non-ischemic myocardium. We did not cross the Doppler flow guide wire and did not measure $\mathrm{CBF}$ distal to the lesion; however, guide wire-induced coronary spasm can occur when the guide wire is located across the lesion. Additionally, total occlusion of the coronary artery was the only direct angiographic evidence of myocardial ischemia.

In summary, we have presented angiographic evidence of the coronary steal phenomenon evoked by intracoronary infusion of BK, an endothelium-dependent coronary vasodilator.

\section{References}

1. Keltz TN, Innerfield M, Gitler B, Cooper JA: Dipyridamole-induced myocardial ischemia. JAMA 1987; 257: 1515-1516

2. Kern MJ, Wolford T, Donohue TJ, Bach RG, Aguirre FV, Caracciolo EA, et al: Quantitative demonstration of dipyridamoleinduced coronary steal and alteration by angioplasty in man: Analysis 
by simultaneous, continuous dual Doppler spectral flow velocity. Cathet Cardiovasc Diagn 1993; 29: 329-334

3. Toda N, Okamura T: Endothelium-dependent and -independent responses to vasoactive substances of isolated human coronary arteries. Am J Physiol 1989; 257: H988-H995

4. Kuga T, Egashira K, Mohri M, Tsutsui H, Harasawa Y, Urabe Y, et al: Bradykinin-induced vasodilation is impaired at the atherosclerotic site but is preserved at the spastic site of human coronary arteries in vivo. Circulation 1995; 92: $183-189$
5. Voudris V, Manginas A, Vassilikos V, Koutelou M, Kantzis J, Cokkinos DV: Coronary flow velocity changes after intravenous dipyridamole infusion: Measurement using intravascular Doppler guide wire. A documentation of flow inhomogeneity. J Am Coll Cardiol 1996; 27: $1148-1155$

6. Egashira K, Katsuda Y, Mohri M, et al: Role of endothelium-derived nitric oxide in coronary vasodilation induced by pacing tachycardia in humans. Circ Res 1996; 79: 331-335 